PROCEEDINGS OF THE

AMERICAN MATHEMATICAL SOCIETY

Volume 132, Number 7, Pages 2171-2181

S 0002-9939(04)07341-1

Article electronically published on February 19, 2004

\title{
ON CLEAVABILITY OF CONTINUA OVER LOTS
}

\author{
RAUSHAN Z. BUZYAKOVA \\ (Communicated by Alan Dow)
}

Abstract. It is shown that any continuum cleavable over a LOTS $L$ is embeddable into $L$.

\section{INTRODUCTION}

This work is devoted to cleavability over LOTS (linearly ordered topological spaces). The general concept of cleavability was introduced by A. V. Arhangelskii (see [Ar2]). Comprehensive surveys on this subject can be found in [Ar5] and [Ar6].

A space $X$ is cleavable over a space $Y$ along $A \subseteq X$ if there exists a continuous mapping $f$ from $X$ to $Y$ such that $f(A) \cap f(X \backslash A)=\emptyset$. A space $X$ is cleavable over a space $Y$ if $X$ is cleavable over $Y$ along each $A \subseteq X$. A space $X$ is said to condense into a space $Y$ if there exists a one-to-one continuous mapping of $X$ into $Y$.

The following are the main problems in this field.

Problem 1.1. When does cleavability of $X$ over $Y$ imply that $X$ embeds (condenses) into $Y$ ?

Problem 1.2. Which properties of $Y$ are possessed by every space cleavable over $Y$ ?

Interesting results related to these problems are obtained in [Ar2], Ar3, Ar4, $[\mathrm{Bal}],[\mathrm{Bu}]$, [Tka], and [Yas]. In this article it will be shown that any continuum cleavable over a LOTS $L$ is embeddable into $L$. (Throughout the paper, all spaces are assumed to be Hausdorff, and by a continuum we mean a connected compact topological space, not necessarily linearly ordered.) Connectedness of a compactum is needed in our theorem, as shown in the following example.

Example 1.3. Let $X, Y$ be three-point and two-point discrete compacta, respectively. It is easy to see that $Y$ is a LOTS and $X$ is cleavable over $Y$. (To cleave $X$ over $Y$ along $A$, just collapse two points that are both in $A$ or its complement.) Obviously, $X$ is not embeddable in $Y$, since $Y$ lacks one extra point. However, $X$ is a LOTS!

Thus, the following question remains of interest.

Received by the editors November 19, 2001 and, in revised form, April 17, 2003.

2000 Mathematics Subject Classification. Primary 54F05, 54F15, 54C25.

Key words and phrases. Cleavability, LOTS, continuum, $\tau$-sequences.

The author gratefully acknowledges the referee's valuable remarks and suggestions.

(C)2004 American Mathematical Society 
Question 1.4 (A. V. Arhangelskii). Let X be a compactum cleavable over a LOTS. Is $X$ a LOTS?

In [Ar3], Arhangelskii answered this question for cleavability over metric spaces. He proved that any compactum cleavable over a metric LOTS is a metric LOTS itself.

For a space $X$ cleavable over a LOTS, a set $A \subset X$ is called absolute for $X$ if any continuous mapping that cleaves $X$ over a LOTS along $A$ is one-to-one. Let $X$ be a LOTS. The character of $x \in X$ from the right, denoted $\chi_{r}(x, X)$, is the smallest cardinal number $\tau$ such that there exists a set $\left\{x_{\alpha}: x_{\alpha}>x, \alpha<\tau\right\}$ whose closure contains $x$. Similarly, we define the character from the left, $\chi_{l}(x, X)$. A $\tau$-sequence is a LOTS homeomorphic to the space of all ordinal numbers less than $\tau$ with the topology of linear order. Note that our definition of $\tau$-sequence is non-standard. An interval of a LOTS is a set in the form $[a, b],(a, b),[a, b)$, or $(a, b]$, where $a \leq b$. If $a=b$, an interval is called trivial. Let $f$ be a continuous mapping of a LOTS $X$. We say that elements $x, y \in X$ are collapsed (glued up) under $f$ if $f(x)=f(y)$. If $I$ is an interval in $X$ and $f(I)$ is a single-element set, we say that $I$ is squeezed into a point under $f$. We will use the following simple known statements.

Statement 1.5. If $X$ is a compact LOTS and $\chi_{l}(x, X)=\tau$, then there exists a $\tau$-sequence converging to $x$ from the left, and $c f(\tau)=\tau$.

Statement 1.6. If $c f(\tau)>\omega_{0}$ and $s_{1}, s_{2}$ are two $\tau$-sequences converging to $x$ from the left, then $s_{1} \cap s_{2} \neq \emptyset$.

We will also use the following obvious fact about absolute sets.

Statement 1.7. If $X$ is a connected infinite LOTS and $A$ is an absolute set for $X$, then $A$ is dense in $X$.

Proof. If $A$ is not dense, then there exists a nontrivial closed interval $I \subset X \backslash$ $A$. Squeeze $I$ into a point. This operation defines a continuous mapping $f$ of $X$ onto a LOTS. Obviously, $f$ cleaves $X$ along $A$, but $f$ is not one-to-one, since $I$ is infinite.

Statement 1.8. If $X=[a, b]$ is a connected infinite LOTS, then there exists a countable $Y \subset X$ such that $|\bar{Y}|=2^{\omega_{0}}$.

Proof. The argument is the same as in the classical construction of the Cantor set. To avoid repetition, let us do only the first two steps. Take arbitrary distinct $y_{1 / 3}, y_{2 / 3} \in(a, b)$. Remove $\left(y_{1 / 3}, y_{2 / 3}\right)$ from $[a, b]$. What is left consists of the two components $\left[a, y_{1 / 3}\right]$ and $\left[y_{2 / 3}, b\right]$. Now take arbitrary distinct $y_{1 / 9}, y_{2 / 9} \in\left(a, y_{1 / 3}\right)$ and $y_{7 / 9}, y_{8 / 9} \in\left(y_{2 / 3}, b\right)$. Again remove central thirds and repeat the abovedescribed process. The set of all end-points of the removed intervals is countable, and its closure has cardinality $2^{\omega_{0}}$.

\section{Some General FACTS}

Proposition 2.1. Let $X=[a, b]$ be a linearly ordered continuum. Let $f$ be $a$ continuous mapping of $X$ onto a LOTS. Suppose that no element in $(a, b)$ collapses with a under $f$. Then, $f(a)$ is an end-point of $f([a, b])$.

Proof. Assume the contrary. Then there exist $x, y \in(a, b]$ such that $f(x)$ and $f(y)$ are the two end-points of $f(X)$. Due to connectedness of $[x, y], f([x, y])=f(X)$. 
Therefore, there exists $z \in(x, y)$ such that $f(z)=f(a)$, which contradicts the proposition's assumptions.

Proposition 2.2. Let $X$ be a connected LOTS. Let $f$ be a continuous non-one-toone mapping of $X$ onto a LOTS. Then:

(1) There exist two nontrivial closed disjoint intervals in $X$ that have the same images under $f$.

(2) Suppose $S$ is a nowhere-dense set in $X$. Then there exist two nontrivial closed disjoint intervals in $X$ that do not intersect $S$ and have the same images under $f$.

Proof. (1) Let $c$ be a point in $X$ that collapses with another point under $f$. Let $C$ be the set of all elements in $X$ that have the same image as $c$. If $C$ contains a nontrivial open interval, then we are done. Otherwise, there exist distinct $a, b \in C$ such that no element in $(a, b)$ collapses with $c$. Then, by Proposition 2.1, $f(a)$ is an end-point of $f([a, b])$ (suppose it is the left end-point). Take $d \in(a, b)$ such that $f(d)$ is the other end-point of $f([a, b])$. Due to connectedness, $f([a, b])=f([a, d])=f([d, b])$. Now, take an arbitrary $z \in(f(a), f(d))$. Let $a_{1} \in(a, d)$ be the closest point to $a$ such that $f\left(a_{1}\right)=z$ and let $b_{1} \in(d, b)$ be the closest point to $b$ such that $f\left(b_{1}\right)=z$. Then, by Proposition 2.1, $f\left(\left[a, a_{1}\right]\right)=f\left(\left[b_{1}, b\right]\right)$.

(2) If $[x, y] \subseteq X \backslash S$ and $f$ is not a homeomorphism on $[x, y]$, then (1) for $X=[x, y]$ gives the required intervals. Thus, we can assume now that $f$ is a homeomorphism on any interval outside of $S$. By (1) there exist two disjoint nontrivial closed intervals $I, J$ such that $f(I)=f(J)$. Take a nontrivial $[a, b] \subseteq I \backslash S$. Now, take a nontrivial $[c, d] \subseteq f^{-1}(f([a, b])) \cap J$ that does not intersect $S$. Thus, $f([c, d]) \subseteq$ $f([a, b])$. Since $f$ is a homeomorphism on both $[a, b]$ and $[c, d]$, there exists $\left[a^{\prime}, b^{\prime}\right] \subseteq$ $[a, b]$ such that $f\left(\left[a^{\prime}, b^{\prime}\right]\right)=f([c, d])$.

Proposition 2.3. Let $f$ be a continuous mapping of a connected LOTS $X$ onto a LOTS. Let $\chi_{l}(x, X)=\tau$. If no nontrivial interval is squeezed into a point under $f$, then either $\chi_{l}(f(x), f(X))=\tau$ or $\chi_{r}(f(x), f(X))=\tau$.

Proof. Since no nontrivial interval is squeezed into a point, there exists a strictly increasing sequence $\left\{x_{\alpha}\right\}$ of cardinality $\tau$ that converges to $x$ from the left such that $f\left(x_{\alpha}\right)<f(x)$ for each $x_{\alpha}$ (or $f\left(x_{\alpha}\right)>f(x)$ ). We will consider only the case when $f\left(x_{\alpha}\right)<f(x)$ for each $x_{\alpha}$. Then, due to continuity of $f, \chi_{l}(f(x), f(X)) \leq \tau$. Suppose $\chi_{l}(f(x), f(X))=\lambda<\tau$. Then there exists a strictly decreasing family of closed intervals $\mathcal{J}=\left\{J_{\alpha}: f(x)\right.$ is the right end-point of $\left.J_{\alpha}, \alpha<\lambda\right\}$ such that $\bigcap \mathcal{J}=\{f(x)\}$. Since $\lambda<c f(\tau), \bigcap f^{-1}(\mathcal{J})$ contains a nontrivial interval of the form $(y, x)$. This interval, by the construction of $\mathcal{J}$, must have the same image as $x$, which contradicts the proposition's assumptions.

Proposition 2.4. Let $f$ be a continuous mapping of a connected LOTS $X$ onto a LOTS. Suppose $x \in X$ is not an end-point of $X$ and $\chi_{l}(x, X)=\tau>\chi_{r}(x, X)=\lambda$. If $f$ squeezes no nontrivial interval into a point, then either $\chi_{l}(f(x), f(X))=\tau>$ $\chi_{r}(f(x), f(X))=\lambda$ or $\chi_{l}(f(x), f(X))=\lambda<\chi_{r}(f(x), f(X))=\tau$.

Proof. Apply Proposition 2.3 twice.

Proposition 2.5. Let $X=[a, b]$ be a linearly ordered continuum with $\chi(a, X)=\tau$ and $\chi(b, X)=\lambda>\tau$. Let $f$ be a continuous mapping of $X$ onto a LOTS such 
that $f(a)=f(b)$. Let $f(a)$ be an end-point of $f(X)$. Then there exists a nontrivial interval in $X$ whose image under $f$ is a single-element set.

Proof. Assume that no nontrivial interval is squeezed into a point. Then, by Proposition 2.3, $\chi(f(a), f(X))=\tau<\chi(f(b), f(X))=\lambda$, which contradicts $f(a)=$ $f(b)$.

Proposition 2.6. Let $f$ be a continuous mapping of a connected LOTS X onto a LOTS. Let $I=[a, b), J=(c, d]$ be two disjoint intervals such that $\left.f\right|_{I},\left.f\right|_{J}$ are embeddings, and $J$ is to the right of $I$. Suppose $f(x)=f(y)$ for $x \in I$ and $y \in J$, and no element in $(x, y)$ collapses with $x$ under $f$. If $f$ preserves (reverses) the order of $I$, then $f$ reverses (preserves) the order of $J$.

Proof. By Proposition 2.1, $f(x)$ is an end-point of $f([x, y])$. If the order of $I$ is preserved, $f(x)$ is the left end-point of $f([x, y])$. Since $J$ is open from the left, there exists $z \in J$ such that $x<z<y$. Since $f(y)$ is the left end-point of $f([x, y])$, $f(z)>f(y)$. Since $\left.f\right|_{J}$ is an embedding and switches the order of two elements, it must reverse all of $J$ (apply connectedness of $J$ ).

If the order of $I$ is reversed, apply the above arguments but interchange "left" by "right", "preserves" by "reverses", etc.

Proposition 2.7. Let $[a, b]$ be a connected LOTS. If $(a, b)$ is first countable, then $\chi_{r}(a,[a, b]) \leq \omega_{1}$ and $\chi_{l}(b,[a, b]) \leq \omega_{1}$.

Proof. If $\chi_{r}(a,[a, b])=\tau>\omega_{1}$, then there exists a $\tau$-sequence converging to $a$ from the right. This sequence contains an $\omega_{1}$-subsequence converging to some element in $(a, b)$, which contradicts first-countability of $(a, b)$.

Proposition 2.8. Let $X=[a, b]$ be a linearly ordered continuum. Let $f$ be $a$ continuous mapping of $X$ onto a LOTS such that $f(a)=f(b)$. Let $c, d$ be elements in $X$ whose images are the two end-points of $f(X)$. Then for any $x \in X \backslash\{c, d\}$ there exists $y \in X \backslash\{x\}$ such that $f(x)=f(y)$.

Proof. We may assume that $|f(X)|>1$ and $c<d$. Since $f(a)=f(b), f([a, c]) \cup$ $f([d, b])=f(X)$. But, $f([c, d])=f(X)$ as well. Thus, any element in $[a, c) \cup(d, b]$ collapses with some element in $[c, d]$. Also, any element in $(c, d)$ collapses with some element in $[a, c] \cup[d, b]$. Since $[a, c) \cup(c, d) \cup(d, b]$ covers all of $X$, except $\{c, d\}$, we are done.

Proposition 2.9. Let $f$ be a continuous one-to-one mapping of a connected LOTS $X$ to a LOTS. Then $f$ is an embedding.

Proof. Since $f$ is a one-to-one continuous mapping of a connected LOTS into a LOTS, $f$ is strictly monotonic. Since $f(X)$ is a connected subspace of a LOTS, it is a LOTS itself. Thus, $f$ is a strictly monotonic mapping of a LOTS $X$ onto a LOTS $f(X)$, and therefore it is a homeomorphism. To see this, notice that images and inverse images of intervals are intervals.

Proposition 2.10. Let $f$ be a continuous mapping of a connected LOTS $X=[a, b]$ to a LOTS. If $f$ is one-to-one on $(a, b)$, then $f$ is an embedding on $[a, b]([a, b))$.

Proof. By Proposition 2.2, $f$ is one-to-one on $[a, b]([a, b))$, and, by Proposition 2.9, $f$ is an embedding on $[a, b]([a, b))$. 


\section{Lemmas}

In our first lemma, we will partially employ a technique developed by Tkachuk Tka].

Lemma 3.1. Let $X$ be a first-countable connected LOTS. Then:

(1) There exists an absolute set $A \subset X$ for $X$.

(2) For two disjoint nowhere dense sets $S_{1}, S_{2} \subset X$, there exists an absolute set $A$ for $X$ that contains $S_{1}$ and does not intersect $S_{2}$.

Proof. It is enough to prove (2). We may assume that $X$ is infinite (if $X$ is a singleelement set, the empty set is absolute for $X$ ). First, notice that the cardinality of $X$ is $2^{\omega_{0}}$. Indeed, any connected LOTS is locally compact. By Proposition 2.7, $X$ can be covered by $\omega_{1}$ many first countable compacta. Now apply Arhangelskii's theorem [Ar1].

Let $\mathcal{J}$ be the set of all nonempty open intervals that have no common elements with $S_{1} \cup S_{2}$. Enumerate $\mathcal{J}=\left\{J_{\alpha}: \alpha<2^{\omega_{0}}\right\}$. Let $\mathcal{P}$ be the set of all possible pairs $(C, D)$, where $C$ and $D$ are countable sets in $X$ whose closures are disjoint and have no common elements with $S_{1} \cup S_{2}$.

For each $(C, D) \in \mathcal{P}$, let $G_{C, D}$ be the set of all one-to-one mappings $g_{C, D}$ from $C$ onto $D$ that possess the following property.

Property $g_{C, D}$. There exist $2^{\omega_{0}}$ mutually disjoint sets $\{c, d\}$, where $c \in \bar{C} \backslash C$ and $d \in \bar{D} \backslash D$, such that for some sequence $\left\{c_{n}: c_{m} \neq c_{k}\right.$ for $\left.m \neq k\right\} \subset C$ we have $c_{n} \rightarrow c$ and $d \in \overline{\left\{g_{C, D}\left(c_{n}\right)\right\}}$.

Enumerate $G=\bigcup_{(C, D) \in \mathcal{P}} G_{C, D}=\left\{g_{\alpha}: \alpha<2^{\omega_{0}}\right\}$. Inductively, we will construct two disjoint sets $A=S_{1} \cup\left\{a_{\alpha}: \alpha<2^{\omega_{0}}\right\} \cup\left\{a_{\alpha}^{\prime}: \alpha<2^{\omega_{0}}\right\}$ and $B=\left\{b_{\alpha}: \alpha<\right.$ $\left.2^{\omega_{0}}\right\} \cup\left\{b_{\alpha}^{\prime}: \alpha<2^{\omega_{0}}\right\}$ with the following two properties.

(*) For every $g_{\alpha}=g_{C, D} \in G$, there exists a sequence $\left\{c_{n}: c_{m} \neq c_{k}\right.$ for $m \neq$ $k\} \subset C$ such that $c_{n} \rightarrow a_{\alpha} \notin C$ and $b_{\alpha} \in \overline{\left\{g_{C, D}\left(c_{n}\right)\right\}} \backslash D$.

$(* *)$ For every $\alpha$, both $a_{\alpha}^{\prime}$ and $b_{\alpha}^{\prime}$ belong to $J_{\alpha}$.

Construction. Assume $a_{\beta}, b_{\beta}$ are defined for $\beta<\alpha<2^{\omega_{0}}$.

Step $\alpha$. Consider $g_{\alpha}=g_{C, D} \in G$. Let $a_{\alpha}$ and $b_{\alpha}$ be any two elements in $X \backslash\left(S_{1} \cup S_{2}\right)$, distinct from all $a_{\beta}, a_{\beta}^{\prime}$ and $b_{\beta}, b_{\beta}^{\prime}$ for $\beta<\alpha$, that play the role of $c$ and $d$ in Property $g_{C, D}$. Also, take arbitrary distinct $a_{\alpha}^{\prime}, b_{\alpha}^{\prime} \in J_{\alpha}$ that are distinct from all $a_{\beta}, b_{\beta}$ for $\beta \leq \alpha$ and from all $a_{\beta}^{\prime}, b_{\beta}^{\prime}$ for $\beta<\alpha$. (This can be done because the set of alreadychosen points has cardinality less than $2^{\omega_{0}}$.)

The set $A$ contains $S_{1}$ by the definition. To see why $A \cap S_{2}=\emptyset$, notice that any element of $A$ is either in $S_{1}$, or $J_{\alpha}$ for some $\alpha$, or $\bar{C}$ for some $(C, D) \in \mathcal{P}$. Every $J_{\alpha}$ has no common elements with $S_{1} \cup S_{2}$ by the definition. By the lemma's assumptions, $S_{1}$ and $S_{2}$ are disjoint. Also, by the definition of $\mathcal{P}$ neither $\bar{C}$ nor $\bar{D}$ has common elements with $S_{2}$.

So now we must show that $A$ is absolute for $X$. Let a continuous mapping $f$ cleave $X$ over a LOTS along $A$. Assume $f$ is not one-to-one. By Proposition 2.2, there exist two disjoint nontrivial closed intervals $I$ and $J$ that do not intersect $S_{1} \cup S_{2}$ and whose images under $f$ coincide. We may assume that both $I$ and $J$ are compacta.

Notice that due to (**), both $A$ and $X \backslash A$ are dense. Since $f(A) \cap f(X \backslash A)=\emptyset$, we have that $|f(I)|=2^{\omega_{0}}$. 
By Statement 1.8, there exists a countable subset $Y \subset f(I)$ with closure of cardinality $2^{\omega_{0}}$. Take countable $C \subset I$ and $D \subset J$ such that $f(C)=f(D)=Y$. We can also require that $f$ is one-to-one on both $C$ and $D$. Define a correspondence $g: C \rightarrow D$ by $g(c)=f^{-1}(f(c)) \cap D$. Since $f$ is one-to-one on both $C$ and $D$ and $f(C)=f(D)=Y, f$ is a well-defined one-to-one function of $C$ onto $D$.

Let us show that $g \in G$. Take any $c \in \bar{C} \backslash C$ such that $f(c) \notin Y$. Since $X$ is first countable, there exists $\left\{c_{n}: c_{m} \neq c_{k}\right.$ for $\left.m \neq k\right\} \subset C$ such that $c_{n} \rightarrow c$. Consider $\left\{g\left(c_{n}\right)\right\}$. Since $f\left(g\left(c_{n}\right)\right)=f\left(c_{n}\right)$ and $f\left(c_{n}\right) \rightarrow f(c)$, there exists $d \in \overline{\left\{g\left(c_{n}\right)\right\}}$ such that $f(d)=f(c)$ (here we use compactness of $J$ ). Since $f(c) \notin Y$, we have that $d \in \bar{D} \backslash D$. To finish verification of Property $g_{C, D}$, notice that there are $2^{\omega_{0}}$ elements in $\bar{C}$ whose images are not in $Y$.

Thus, $g=g_{\alpha} \in G$ for some $\alpha$. Therefore, there exists a sequence $\left\{c_{n}: c_{m} \neq\right.$ $c_{k}$ for $\left.m \neq k\right\} \subset C$ such that $c_{n} \rightarrow a_{\alpha} \notin C$ and $b_{\alpha} \in \overline{\left\{g\left(c_{n}\right)\right\}} \backslash D$. By the definition of $g, f\left(b_{\alpha}\right) \in \overline{\left\{f\left(c_{n}\right)\right\}}$. Since $f$ is one-to-one on $D, f\left(b_{\alpha}\right)$ is an accumulation point of $\left\{f\left(c_{n}\right)\right\}$. But the latter set has only one accumulation point, namely $f(c)$, since $\left\{f\left(c_{n}\right)\right\}$ is a non-constant convergent sequence. Therefore, $f\left(a_{\alpha}\right)=f\left(b_{\alpha}\right)$, which contradicts the choice of $f$. This concludes the proof of Lemma 3.1.

Lemma 3.2. Let $X$ be a linearly ordered continuum with an open dense set of points of countable character. Then there exists an absolute set $A \subset X$ for $X$.

Proof. We say that a set $O$ is a countable character component if it is a maximal open interval containing points only of countable character. Let $\mathcal{O}=\left\{O_{\alpha}\right\}$ be the maximal family of mutually disjoint countable character components. Obviously, $\cup \mathcal{O}$ is open and dense in $X$. For each $O_{\alpha} \in \mathcal{O}$, we perform the following steps 1 and 2 .

1. Let $a_{\alpha}, b_{\alpha}$ be the left and right end-points of $\bar{O}_{\alpha}$. By Proposition 2.7, $\chi_{r}\left(a_{\alpha}, \bar{O}_{\alpha}\right)$ does not exceed $\omega_{1}$. If $\chi_{r}\left(a_{\alpha}, \bar{O}_{\alpha}\right)=\omega_{1}$, fix an $\omega_{1}$-sequence $r_{\alpha} \subset$ $O_{\alpha} \backslash\left\{b_{\alpha}\right\}$ converging to $a_{\alpha}$ from the right. Similarly, if $\chi_{l}\left(b_{\alpha}, \bar{O}_{\alpha}\right)=\omega_{1}$, fix an $\omega_{1}$-sequence $l_{\alpha} \subset O_{\alpha} \backslash \bar{r}_{\alpha}$ converging to $b_{\alpha}$ from the left. If $\chi_{r}\left(a_{\alpha}, \bar{O}_{\alpha}\right)<\omega_{1}$, let $r_{\alpha}=\emptyset$. Similarly, if $\chi_{l}\left(b_{\alpha}, \bar{O}_{\alpha}\right)<\omega_{1}$, let $l_{\alpha}=\emptyset$.

2. Fix an absolute for $O_{\alpha}$ set $A_{\alpha} \subset O_{\alpha}$ that does not intersect $l_{\alpha}$ but contains $r_{\alpha}$ (Lemma 3.1).

Define a set $B$ as follows: $b \in B$ if and only if $b$ is the right end-point for some $O \in \mathcal{O}$, and is an accumulation point for the family $\mathcal{O}$ (i.e., a limit point of $\bigcup \mathcal{O}$ that is not a limit point of any single element of $\mathcal{O}$ ). Let us show that $A=\bigcup\left\{A_{\alpha}\right\} \cup B$ is an absolute set for $X$.

Let a continuous mapping $f$ cleave $X$ over a LOTS along $A$. Assume $f$ is not oneto-one. By the choice of $A,\left.f\right|_{O}$ is one-to-one for any $O \in \mathcal{O}$. Also, by Proposition 2.10, $\left.f\right|_{O}$ is an embedding.

By Proposition 2.2 and density of $\bigcup \mathcal{O}$, there exists $x \in A_{\alpha} \subset O_{\alpha} \in \mathcal{O}$ that collapses with some element from the right. Let $y=\inf \{z \in X: z>x, f(z)=$ $f(x)\}$. Since $O_{\alpha}$ is open and $f$ is one-to-one on $O_{\alpha}, y$ is strictly greater than $x$. By the choice of $f, y \in O_{\beta} \in \mathcal{O}$ or $y$ is the right end-point of $O_{\beta}$ for some $\beta \neq \alpha$. Assume $f$ preserves the order of $O_{\alpha}$. By Proposition 2.6, $f$ reverses $O_{\beta}$.

Since $f$ is one-to-one on each $O \in \mathcal{O}$, no nontrivial interval is squeezed into a point. Also, by Proposition 2.3, $f$ preserves the character of each non-end-point of $X$. Since any neighborhood of an end-point of $O_{\alpha}$ or $O_{\beta}$ contains points of uncountable character, $b_{\alpha}\left(a_{\beta}\right)$ cannot collapse with any element in $O_{\beta}\left(O_{\alpha}\right)$. Thus, 
$f\left(b_{\alpha}\right)=f\left(a_{\beta}\right)$. If $b_{\alpha}$ is an accumulation point for the family $\mathcal{O}$ then, by the latter equality, $f(A)$ intersects $f(X \backslash A)$, which contradicts the choice of $f$ (recall that $a_{\beta}$ is the left end-point of $O_{\beta}$ and, therefore, cannot be an accumulation point for $\mathcal{O}$ and the right end-point for some $O$ at the same time). If $b_{\alpha}$ is not an accumulation point, then it is the boundary point of two elements in $\mathcal{O}$, and, due to maximality of each $O$, cannot be of countable character. Therefore, there exists an $\omega_{1}$-sequence (see Proposition 2.7) that converges to $b_{\alpha}$ from the right or left. Assume this sequence is $l_{\alpha}$. Since $f$ reverses $O_{\beta}$ and preserves characters, there exists an $\omega_{1}$-sequence $r_{\beta}$ that converges to $a_{\beta}$ from the right. Obviously, under $f$, images of these sequences intersect. But $l_{\alpha}$ is not in $A$ while $r_{\beta}$ is in $A$. A similar argument works if the $\omega_{1}$-sequence (some $r_{\gamma}$ ) converges to $b_{\alpha}$ from the right. This contradiction implies that $f$ is one-to-one.

Lemma 3.3. Let $X$ be a connected LOTS, and let $A$ be a dense set that does not contain end-points and possesses the following property.

$\left(^{*}\right) \chi_{l}(x, X)<\chi_{r}(x, X)$ for all $x \in A$.

Then:

(1) The set $A$ is absolute for $X$.

(2) A set $A^{+}$which is the union of $A$ and an arbitrary discrete-in-itself set of elements of countable character in $X$ is absolute for $X$ as well.

Proof. It is enough to prove (2) only. Let a continuous mapping $f$ cleave $X$ over a LOTS along $A^{+}$. Assume $f$ is not one-to-one. First, notice that no nontrivial open interval $I$ collapses into a point. Indeed, there is a decreasing $\omega_{0}$-sequence converging to $x \in I$. Since $x$ does not satisfy $(*)$, it is not in $A$. The set of such $x$ 's is dense, while $A^{+} \backslash A$ is not. Therefore, each nontrivial open interval contains elements from both $A^{+}$and its complement.

By Proposition 2.2, there are two disjoint nontrivial intervals in $X$ whose images coincide. Since $A$ is dense, there exists $a \in A$ that is glued up under $f$ with an element to the right of $a$. Let $b=\inf \{x \in X: x>a, f(x)=f(a)\}$. Then $b \geq a$.

If $b=a$, then there exists a decreasing $\tau$-sequence $\left\{x_{\alpha}: f\left(x_{\alpha}\right)=f(a), \alpha<\tau\right\}$ that converges to $a$ from the right and $\tau>\omega_{0}$ (see $\left.(*)\right)$. Since $\tau>\omega_{0}$, the $\tau$ sequence contains an $\omega_{0}$-sequence $\left\{y_{1}=x_{\omega_{0}}, y_{2}=x_{\omega_{0}+\omega_{0}}, \ldots\right\}$ that converges to $y=x_{\omega_{0}^{2}}$. Because of right-cofinality, no term $y_{n}$ can satisfy $(*)$, and, therefore, is not in $A$. For the same reason, $y \notin A$ either. Since $A^{+} \backslash A$ is discrete in itself, it cannot contain all $y_{n}$ 's and $y$. So, either some $y_{n}$ or $y$ does not belong to $A^{+}$. But that is impossible, because $f\left(y_{n}\right)=f(y)=f(a)$ and $f$ cleaves $X$ along $A^{+}$.

If $b>a$, then, due to the choice of $f, b \in A^{+}$. By Proposition 2.1, $f(b)$ is an end-point of $f([a, b])$. Also, by Proposition 2.5, $\chi_{r}(a, X)=\chi_{l}(b, X)$. Then, $b \in A$. By Proposition 2.4, $\chi_{l}(a, X)=\chi_{r}(b, X)$. As a result of these two equalities, $\chi_{l}(b, X)>\chi_{r}(b, X)$, which contradicts $(*)$.

Lemma 3.4. Let $X$ be a connected LOTS with the following properties:

1. There exists an open dense $O \subseteq X$ such that $\chi_{l}(x, X)=\chi_{r}(x, X)$ for all $x \in O$.

2. The set of elements of uncountable character is dense in $X$.

Then:

(1) There exists a set $A \subset O$ with the following properties:

A1. The set of all $x \in A$ with $\chi_{l}(x, X)=\chi_{r}(x, X)=\omega_{1}$ is dense in $X$.

A2. All $x$ in $A$ are of character either $\omega_{0}$ or $\omega_{1}$. 
A3. For each $x \in A$ of character $\omega_{1}$ there exist an $\omega_{1}$-sequence $r_{x} \subset A$ converging to $x$ from the right and an $\omega_{1}$-sequence $l_{x} \subset X \backslash A$ converging to $x$ from the left.

(2) The set $A$ in (1) is absolute for $X$.

Proof. (1) First, notice that any nonempty open interval $I$ in $X$ contains an $x$ with $\chi_{l}(x, X)=\chi_{r}(x, X)=\omega_{1}\left(\omega_{0}\right)$. Indeed, due to property 2 and density of $O, I$ contains a $\tau$-sequence $\left(\tau>\omega_{0}\right)$ whose closure is in $O \cap I$. This sequence contains an $\omega_{1}\left(\omega_{0}\right)$-subsequence converging to some $x \in I \cap O$ from the right (left). Then $\chi_{r}(x, X)=\omega_{1}\left(\omega_{0}\right)$. Due to the definition of $O$ we have the required equality.

Let $a, b$ be the end-points of a linear ordered compactification of $X$. Inductively, we will define the sets $A_{\alpha}$ and $B_{\alpha}$ such that $A=\bigcup A_{\alpha}$ and the set $B=\bigcup B_{\alpha}$ contains all the $l_{x}$ 's defined in property $A 3$.

Suppose $A_{\beta}$ and $B_{\beta}$ are defined for all $\beta<\alpha$.

For each pair $\{c, d\}$, where $c<d$, satisfying the following requirements 1, 2:

1. $\{c, d\} \in\left(\overline{\bigcup_{\beta<\alpha}\left(A_{\beta} \cup B_{\beta}\right)} \cup\{a, b\}\right)$,

2. $(c, d) \cap\left(\overline{\bigcup_{\beta<\alpha}\left(A_{\beta} \cup B_{\beta}\right)} \cup\{a, b\}\right)=\emptyset$ (that is, $c, d$ are neighbors),

pick up an arbitrary point $x \in(c, d) \cap O$ of character $\omega_{1}$. Choose two disjoint $\omega_{1}$ sequences $r_{x}, l_{x} \subset(c, d) \cap O$ converging to $x$ from the right and left, respectively, and consisting of points of countable character.

If no such $c, d$ exist, construction stops.

Let $A_{\alpha}=\left(\bigcup_{\beta<\alpha} A_{\beta}\right) \cup\left(\bigcup\left\{\right.\right.$ all $\left.\left.\overline{r_{x}}\right\}\right)$ and $B_{\alpha}=\left(\bigcup_{\beta<\alpha} B_{\beta}\right) \cup\left(\bigcup\left\{\right.\right.$ all $\left.\left.l_{x}\right\}\right)$.

Let $A=\bigcup A_{\alpha}$ and $B=\bigcup B_{\alpha}$. Properties $A 2, A 3$ as well as the property of $B$ follow from the construction. Let us show that $A$ possesses property $A 1$.

Take an arbitrary nonempty open interval $J$. Suppose, $J \cap(A \cup B)=\emptyset$. Take $c \in \overline{A \cup B \cup\{a\}}$ the closest to $J$ from the left and $d \in \overline{A \cup B \cup\{b\}}$ the closest to $J$ from the right. The pair $\{c, d\}$ satisfies the construction requirements, and therefore, construction must continue. Thus, $A \cup B$ is dense. During construction, from each interval we pick up not more than a nowhere dense set of elements for $A_{\alpha}$ and $B_{\alpha}$. Once we pick up at least two points from $J$, at the next step there exists a pair $\{c, d\}$ satisfying the construction requirements. At that step we pick up an $x \in(c, d)$ for $A$ with $\chi_{l}(x, X)=\chi_{r}(x, X)=\omega_{1}$.

(2) Now, let us show that $A$ is absolute for $X$. Let $f$ cleave $X$ over a LOTS along $A$. Assume $f$ is not one-to-one. Due to $A 1$ and Proposition 2.2, there exists $x \in A$ with $\chi_{l}(x, X)=\chi_{r}(x, X)=\omega_{1}$ that collapses with some element from its left side. Let $y$ be the closest point from the left of $x$ that has the same image as $x$.

If no such $y$ exists, then some $\omega_{1}$-sequence converging to $x$ from the left, and, therefore, the most part of $l_{x}$ are squeezed into $f(x)$. But $l_{x}$ is not in $A$, while $x$ is.

If $y$ exists, then $y \in A$ (by the choice of $f$ ). Suppose $y$ is of countable character. By Proposition 2.1, $f(x)$ is an end-point of $f([y, x])$. Then, by Proposition 2.5, there exists an interval that is squeezed into a point under $f$. But any interval contains elements from both $A$ and its complement (this follows from the definition of $B$ and property $A 1$ ). If $y$ is of character $\omega_{1}$, then the images of $r_{y}$ and $l_{x}$ intersect (recall that $r_{y}, l_{x}$ are both $\omega_{1}$-sequences). But $r_{y} \subset A$ while $l_{x} \subset B \subset X \backslash A$. Since all the possibilities bring us to contradictions, we conclude that $f$ is one-to-one. 


\section{THEOREM}

Theorem 4.1. Let $X$ be a linearly ordered continuum cleavable over a LOTS L. Then, $X$ is embeddable to $L$.

Proof. Let us define families $\mathcal{B}, \mathcal{C}, \mathcal{D}$, and $\mathcal{E}$ as follows. Family $\mathcal{B}$ consists of all maximal nontrivial closed intervals in $X$ containing an open dense set of elements of countable character in $X$. Family $\mathcal{C}$ consists of all maximal open intervals in $X$ containing a dense set of elements $x$ with $\chi_{l}(x, X)>\chi_{r}(x, X)$. Family $\mathcal{D}$ consists of all maximal open intervals in $X \backslash \overline{\cup \mathcal{C}}$ containing a dense set of elements $x$ with $\chi_{l}(x, X)<\chi_{r}(x, X)$.

Family $\mathcal{D}$ possesses the following properties:

1. For each $D \in \mathcal{D}$, the set of all elements $x$ with $\chi_{l}(x, X)>\chi_{r}(x, X)$ is nowhere-dense in $D$.

This follows from the maximality of Family $\mathcal{C}$.

2. Each $D \in \mathcal{D}$ contains a dense set of elements of countable character.

Indeed, take any open interval and a decreasing countable sequence inside the interval. Let the sequence converge to $x$. Due to property 1 , we can pick up a sequence in such a manner that $\chi_{l}(x, X) \leq \chi_{r}(x, X)=\omega_{0}$.

Family $\mathcal{E}$ consists of all maximal open intervals in $X \backslash \overline{\cup(\mathcal{B} \cup \mathcal{C} \cup \mathcal{D})}$.

Family $\mathcal{E}$ possesses the following property.

Each $E \in \mathcal{E}$ contains an open dense set of elements $x$ with $\chi_{l}(x, X)=\chi_{r}(x, X)$, and a dense set of elements of uncountable character.

This follows from the maximality of the above families.

Let $\mathcal{S}=\mathcal{B} \cup \mathcal{C} \cup \mathcal{D} \cup \mathcal{E}$. First, notice that elements in $\mathcal{S}$ are disjoint due to maximality of each family, and their union is dense in $X$. Also, the closures of two elements from the same family are disjoint.

For each element $B \in \mathcal{B}, C \in \mathcal{C}$, and $E \in \mathcal{E}$ fix absolute sets $A_{B}, A_{C}, A_{E}$ in accordance with Lemma 3.2, Lemma 3.3 (1), and Lemma 3.4 (2).

For each $D \in \mathcal{D}$, fix an absolute set $A_{D}$ from Lemma $3.3(2)$, so that $A_{D}$ contains a discrete-in-itself set of points of countable character accumulating to endpoints of $\bar{D}$. This can be done, thanks to property 2 of family $\mathcal{D}$.

Let $A=\bigcup\left\{A_{S}: S \in \mathcal{S}\right\}$. Let us show that any continuous mapping $f$ that cleaves $X$ over $L$ along $A$ is an embedding.

By the choice of each $A_{S},\left.f\right|_{S}$ is one-to-one for each $S \in \mathcal{S}$. Also, by Proposition $2.10,\left.f\right|_{S}$ is an embedding.

Suppose $f$ is not one-to-one. Then there exist two sets $S_{1}, S_{2}$ whose images intersect. This follows from the density of $\bigcup \mathcal{S}$ and Proposition 2.2.

We can assume that $S_{1}$ is not from $\mathcal{B}$. Indeed, let $x \in B \in \mathcal{B}$ collapse with some element. Take $y$ from the right (left) of $x$ that has the same image as $x$. By Proposition 2.8, any element in $[x, y]$, except maybe two points, is glued up with some other element. Since $\left.f\right|_{B}$ is one-to-one, $[x, y] \backslash B$ is a nontrivial interval. Since no two elements of $\mathcal{B}$ are neighbors, there exists $S_{1} \in \mathcal{S} \backslash \mathcal{B}$ such that $S_{1} \cap(x, y) \neq \emptyset$ (recall that each $S \in \mathcal{S} \backslash \mathcal{B}$ is open). Fix this $S_{1}$.

Without loss of generality, $f$ preserves the order of $S_{1}$. There exists $S_{2}$ whose image intersects the image of $S_{1}$ by a nontrivial interval and $f$ reverses the order of $S_{2}$. Indeed, let $x \in A_{S_{1}}$ collapse with some element (such an $x$ exists, since $A_{S_{1}}$ is dense in $S_{1}$ ). Let $y$ be the closest element to $x$ from the right (left) such that $f(x)=f(y)$ (such a $y$ exists, since $S_{1}$ is open and $f$ is one-to-one on $S_{1}$ ). By the 
choice of $f, y$ belongs to some $S_{2} \in \mathcal{S}$. Since $S_{1}$ is open, $f\left(S_{1}\right) \cap f\left(S_{2}\right)$ contains an interval. This means that $S_{2}$ cannot be from $\mathcal{B}$, since no interval of $S_{1}$ is first countable. Thus, $S_{2} \in \mathcal{S} \backslash \mathcal{B}$. By Proposition 2.6, $f$ reverses $S_{2}$. Fix this $S_{2}$.

Now, we need only to show that the rest of the possible choices for $S_{1}$ and $S_{2}$ lead to contradictions.

Case 1. $S_{1} \in \mathcal{E}, S_{2} \in \mathcal{C} \cup \mathcal{D}$. As mentioned earlier, $\left.f\right|_{S_{1}}$ and $\left.f\right|_{S_{2}}$ are embeddings. But no open interval of $S_{1}$ is homeomorphic to any open part of $S_{2}$. This follows from the definitions of the families.

Case 2. $S_{1}, S_{2} \in \mathcal{C}$. Due to density of the absolute set, there exist $a_{1} \in A_{S_{1}}$ and $a_{2} \in A_{S_{2}}$ with $f\left(a_{1}\right)=f\left(a_{2}\right)$. Since $f$ preserves the order of $S_{1}, \chi_{l}\left(f\left(a_{1}\right), f(X)\right)>$ $\chi_{r}\left(f\left(a_{1}\right), f(X)\right)$. Since the order of $S_{2}$ is reversed, $\chi_{l}\left(f\left(a_{2}\right), f(X)\right)<\chi_{r}\left(f\left(a_{2}\right)\right.$, $f(X))$. These two inequalities contradict $f\left(a_{1}\right)=f\left(a_{2}\right)$. (For the inequalities, see Lemma $3.3,(1)$.)

Case 3. $S_{1}, S_{2} \in \mathcal{D}$. The argument is similar to Case 2 .

Case 4. $S_{1}, S_{2} \in \mathcal{E}$. Due to density of the absolute set, there exist $a_{1} \in A_{S_{1}}$ and $a_{2} \in A_{S_{2}}$ such that $f\left(a_{1}\right)=f\left(a_{2}\right)$ and $\chi_{l}\left(f\left(a_{i}\right), f(X)\right)=\chi_{r}\left(f\left(a_{i}\right), f(X)\right)=\omega_{1}$ (see Lemma 3.4, (1), Property A1). Suppose $a_{1}<a_{2}$. Since the order of $S_{1}$ is preserved while that of $S_{2}$ is reversed, $f\left(r_{a_{2}}\right) \cap f\left(l_{a_{1}}\right) \neq \emptyset$ (recall that $r_{a_{2}}, l_{a_{1}}$ are $\omega_{1}$-sequences). But $r_{a_{2}}$ is in $A$ while $l_{a_{1}}$ is in the complement of $A$, which contradicts the choice of $f$ (see Lemma 3.4, (1), Property A3).

Case 5. $S_{1} \in \mathcal{D}, S_{2} \in \mathcal{C}$. Assume $S_{2}$ is to the right of $S_{1}$. By the choice of $S_{1}, S_{2}$, there exist $s_{1} \in S_{1}$ and $s_{2} \in S_{2}$ such that $f\left(s_{1}\right)=f\left(s_{2}\right)$. By Proposition 2.8 , any element in $\left[s_{1}, s_{2}\right]$, except maybe two points, collapses with some other element. Since the right end-point of $\overline{S_{1}}$ is in $\left[s_{1}, s_{2}\right]$, there exists $a_{1} \in A_{S_{1}} \cap\left[s_{1}, s_{2}\right]$ of countable character that collapses with some other element (see the definition of $\left.A_{D}\right)$. Take $a_{2}$ the closest to $a_{1}$ from the right (left) such that $f\left(a_{1}\right)=f\left(a_{2}\right)$. Such an $a_{2}$ exists, since $S_{1}$ is open and $f$ is an embedding on $S_{1}$. By the choice of $f, a_{2}$ must belong to $A_{S_{3}}$ for some $S_{3} \in \mathcal{S}$. By Proposition 2.6, $f$ reverses $S_{3}$. Since $a_{1}$ is of countable character, $a_{2}$ must be of countable character as well. Therefore, $S_{3}$ is not in $\mathcal{C}$. The other cases have already been eliminated.

Thus, $f$ is one-to-one, and, therefore, an embedding (recall that $f$ is from a compactum).

Theorem 4.2. Any continuum $X$ cleavable over a LOTS $L$ is embeddable into L.

Proof. By the theorem in $[\mathrm{Bu}$, any continuum cleavable over a LOTS is a LOTS itself. Now, apply Theorem 4.1.

\section{REFERENCES}

[Ar1] A. V. Arhangelskii, The power of bicompacta with first axiom of countability, Dokl. Akad. Nauk SSSR, 187 (1969), 967-970 (in Russian); English transl., Soviet Math. Dokl. 10 (1969), 951-955. MR 40:4922

[Ar2] A. V. Arhangelskii, The general concept of cleavability of a topological space, Topology Appl., 44 (1992), 27-36. MR 93k:54047

[Ar3] A. V. Arhangelskii, Cleavability over reals, Topology Appl., 48 (1992), 163-178. MR 94a:54041

[Ar4] A. V. Arhangelskii, On cleavability of topological spaces over $R, R^{n}$, and $R^{\omega}$, Ann. New York Acad. Sci., 659 (1992), 18-28. MR 98g:54008

[Ar5] A. V. Arhangelskii, A survey of cleavability, Topology Appl., 54 (1993), 141-163. MR 95c:54015 
[Ar6] A. V. Arhangelskii, Some recent achievements and open problems in general topology, Russian Math. Surveys, 52:5(1997), 929-953. MR 99b:54001

[Bal] Z. Balogh, There is a paracompact Q-set space in ZFC, Proc. Amer. Math. Soc., 126:6 (1998), 1827-1833. MR 98g:54084

[Bu] R. Z. Buzyakova, Splittability of compacta over linearly ordered spaces and over the class of all linearly ordered spaces, Moscow Univ. Math. Bull., 51:3 (1996), 44-45. MR 98b:54044

[Tka] V. V. Tkachuk, A note on splittable spaces, Comment. Math. Univ. Carolinae, 33(1992), 551-555. MR 94a:54071

[Yas] I. V. Yashchenko, Embeddings into $R^{n}$ and $R^{\omega}$ and cleavability, Moscow Univ. Math. Bull., 47:2(1992).

[Eng] R. Engelking, General Topology, Sigma Series in Pure Mathematics, 6, Heldermann, Berlin, revised ed., 1989. MR 91c:54001

Department of Mathematics, Brooklyn College, Brooklyn, New York 11210

E-mail address: RaushanB@brooklyn.cuny.edu 